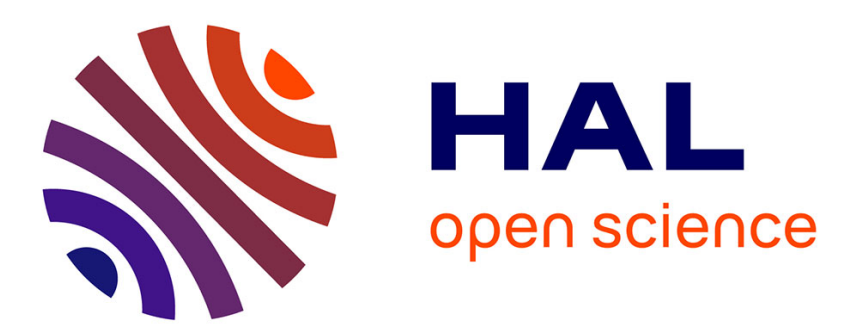

\title{
Relating Lagrangian passive scalar scaling exponents to Eulerian scaling exponents in turbulence
}

François G Schmitt

\section{To cite this version:}

François G Schmitt. Relating Lagrangian passive scalar scaling exponents to Eulerian scaling exponents in turbulence. The European Physical Journal B: Condensed Matter and Complex Systems, 2005, 48, pp.129-137. 10.1140/epjb/e2005-00374-1 . hal-00111906

\section{HAL Id: hal-00111906 \\ https://hal.science/hal-00111906}

Submitted on 17 Mar 2008

HAL is a multi-disciplinary open access archive for the deposit and dissemination of scientific research documents, whether they are published or not. The documents may come from teaching and research institutions in France or abroad, or from public or private research centers.
L'archive ouverte pluridisciplinaire HAL, est destinée au dépôt et à la diffusion de documents scientifiques de niveau recherche, publiés ou non, émanant des établissements d'enseignement et de recherche français ou étrangers, des laboratoires publics ou privés. 


\title{
Relating Lagrangian passive scalar scaling exponents to Eulerian scaling exponents in turbulence
}

\author{
François G. Schmitt \\ CNRS, UMR 8013 ELICO, Wimereux Marine Station, University of Lille 1 \\ 28 av. Foch, 62930 Wimereux, France
}

\begin{abstract}
Intermittency is a basic feature of fully developed turbulence, for both velocity and passive scalars. Intermittency is classically characterized by Eulerian scaling exponent of structure functions. The same approach can be used in a Lagrangian framework to characterize the temporal intermittency of the velocity and passive scalar concentration of a an element of fluid advected by a turbulent intermittent field. Here we focus on Lagrangian passive scalar scaling exponents, and discuss their possible links with Eulerian passive scalar and mixed velocity-passive scalar structure functions. We provide different transformations between these scaling exponents, associated to different transformations linking space and time scales. We obtain four new explicit relations. Experimental data are needed to test these predictions for Lagrangian passive scalar scaling exponents.
\end{abstract}

Key words: Turbulence, Intermittency, Scaling, Multifractal PACS: 47.27.Gs., 47.53.+n

\section{Introduction: Scaling laws for Eulerian and Lagrangian turbu- lence}

A better description, understanding and prediction of the properties of the mixing of passive scalars by a turbulent flow is important for many applications including oceanic and atmospheric dispersion studies, chemical engineering and combustion. In fully developed turbulence, interesting properties of these flows are scale invariance and intermittency for velocity and passive scalars. We consider here the inertial convective subrange, associated to large Peclet and Reynolds numbers; we do not consider the viscous convective range, and thus we can assume that the Prandtl (or Schmidt) number $\left(=\nu / \Gamma_{\theta}\right.$ where $\nu$ is 
the viscosity and $\Gamma_{\theta}$ is the scalar diffusivity of the fluid) is of order 1 . However, even for larger Prandtl numbers, inertial range characteristics are not expected to be modified [1] and the results obtained here should apply as well. Turbulent properties can be considered in an Eulerian or Lagrangian framework, leading to quite different scaling laws. Here we focus on the Lagrangian approach, which is important for transport and mixing studies; it is also the natural description for stochastic models of turbulent mixing (see [2]).

Since passive scalars are advected by the velocity field, we recall here the scaling laws for the velocity as well, but the focus of the paper are passive scalar scaling laws. For scales belonging to the inertial convective range, denoting $\Delta U_{\ell}=|U(x+\ell)-U(x)|$ and $\Delta \theta_{\ell}=|\theta(x+\ell)-\theta(x)|$ the longitudinal increments of the Eulerian velocity and passive scalar fields at a spatial scale $\ell$, we have Kolmogorov's law for the velocity [3]

$$
\Delta U_{\ell} \sim \epsilon^{1 / 3} \ell^{1 / 3}
$$

and Obukhov-Corrsin's law for a passive scalar $[4,5]$

$$
\Delta \theta_{\ell} \sim \epsilon^{-1 / 6} \chi^{1 / 3} \ell^{1 / 3}
$$

where $\epsilon$ is the dissipation, $\chi=\Gamma_{\theta}\left\langle|\nabla \theta|^{2}\right\rangle$ is the scalar variance dissipation rate and $\Gamma_{\theta}$ is the scalar diffusivity of the fluid.

Concerning Lagrangian fields, let us note $V\left(x_{0}, t\right)$ and $\Theta\left(x_{0}, t\right)$ the velocity and passive scalar concentration of an element of fluid at time $t$, initially at a position $x(0)=x_{0}$. In the following we note these $V(t)$ and $\Theta(t)$ since we assume statistical homogeneity. For the passive scalar case, let us recall that a contaminant with zero diffusivity will have the property that each moving fluid particle's contaminant concentration remains equal to its value at the time of release, hence the Lagrangian passive scalar fluctuations do not exist (see [6], p. 236). However, when there is a non-zero molecular diffusivity, local values of the velocity strain rate interact with small-scale inhomogeneities of the passive scalar concentration, leading to exponentially dumped inhomogeneities [6]. The molecular diffusivity of the scalar has thus large scale effects, producing non-trivial fluctuations of passive scalars even in Lagrangian coordinates.

For Lagrangian statistics, scaling laws have been obtained dimensionnally along the same lines as for the Eulerian framework, for the Lagrangian velocity and passive scalar time increments $\Delta V_{\tau}=|V(t+\tau)-V(t)|$ and $\Delta \Theta_{\tau}=$ $|\Theta(t+\tau)-\Theta(t)|$. This gives Landau's relation for the velocity [7]

$$
\Delta V_{\tau} \sim \epsilon^{1 / 2} \tau^{1 / 2}
$$

and Inoue's law for passive scalars [8]

$$
\Delta \Theta_{\tau} \sim \chi^{1 / 2} \tau^{1 / 2}
$$


We may note that the Eulerian power spectra are of the form $E(k) \sim k^{-5 / 3}$ for velocity and passive scalars ( $k$ is the wave number), whereas for Lagrangian fields, the power spectra are also scaling, with a different exponent: $E(f) \sim$ $f^{-2}$ for both velocity and passive scalars ( $f$ is the frequency).

These laws provide velocity and passive scalar fluctuations in time or in space, assuming constant and homogeneous values for the fields $\epsilon$ and $\chi$. In fact, one of the characteristic features of fully developed turbulence is the intermittent nature of the fluctuations of associated fields, providing intermittent corrections for Eulerian and Lagrangian fields (see reviews in [9-11]). This is discussed in the next section, with the introduction of the structure function's framework and mixed structure functions for Eulerian velocity and passive scalars. The following section presents several hypotheses to relate Lagrangian passive scalar scaling exponents to Eulerian passive scalar exponents and mixed velocity-passive scalar exponents; four predictions are obtained and are compared to each other in the last section.

\section{Eulerian and Lagrangian intermittency}

\subsection{Eulerian case}

For Eulerian turbulence, intermittency provides corrections to Kolmogorov and Obukhov-Corrsin (KOC hereafter) scaling laws, which are now well established and received considerable attention in the last twenty years. In the inertial range, the fluctuations of $\Delta U_{\ell}$ and $\Delta \theta_{\ell}$ are characterized using the scale invariant moment functions $\zeta_{u}(q)$ and $\zeta_{\theta}(q)$ (see e. g. [9-12] for a review):

$$
\left\langle\left(\Delta U_{\ell}\right)^{q}\right\rangle \sim \ell^{\zeta_{u}(q)} ;\left\langle\left(\Delta \theta_{\ell}\right)^{q}\right\rangle \sim \ell^{\zeta_{\theta}(q)}
$$

where $q$ is the order of moment. KOC's initial proposals, for non-intermittent dissipation fields, lead to $\zeta_{u}(q)=q / 3$ and $\zeta_{\theta}(q)=q / 3$. For intermittent turbulence, these are cumulant generating function, and are nonlinear and concave. In Eqs. (1) and (2), the dissipation fields $\epsilon$ and $\chi$ are then replaced by random fluxes at scale $\ell, \epsilon_{\ell}$ and $\chi_{\ell}$. For the velocity, only the third moment has no intermittency correction $\left(\zeta_{u}(3)=1\right)$, whereas for the passive scalar, there is no such exact relation, due to the nonlinear product of two fluxes $\epsilon_{\ell}$ and $\chi_{\ell}$ in Eq.(2). Let us note that in the framework of Kolmogorov's hypothesis, the small scale statistics are assumed to be universal, and hence the scaling exponents $\zeta_{u}$ and $\zeta_{\theta}$ are expected to be rather stable and independent of the Reynolds number and of the type of large scale flow. Indeed, for the velocity field, many experimental and Direct Numerical Simulation (DNS) studies have been performed, and the results obtained are in favor of this universality for moments up to about 7 [13]. For passive scalar scaling exponents (for a 
review see [1]) let us mention the important result indicating that even in case of uncorrelated velocity field, the passive scalar field is multiscaling (see [14], and [15] for a review). However, such scaling exponents are quite far from experimental estimates, indicating that intermittency in velocity fluctuations has influence on temperature scaling exponents.

Many experimental and numerical studies have reported estimations of $\zeta_{\theta}(q)$ : Figure 1 shows some of these curves, published in Refs. [16-23]. This shows a rather good stability of $\zeta_{\theta}(q)$ for $q \leq 4$. Since we need below an acceptable average $\zeta_{\theta}$ curve, we estimated averaged values of published estimates of $\zeta_{\theta}(q)$ for integer values of $q$ from 1 to 8. This is shown in Fig. 1 as a continuous curve; the corresponding values are given in Table 1, together with an error bar corresponding to various experimental estimates.

Let us also introduce the mixed velocity-passive scalar structure functions, originally proposed by Antonia and van Atta [24]:

$$
\left\langle\Delta \theta_{\ell}^{p} \Delta U_{\ell}^{q}\right\rangle \sim \ell^{z(p, q)}
$$

where $z(p, q)$ are the associated scaling exponents, with the obvious property $z(0, q)=\zeta_{u}(q)$ and $z(p, 0)=\zeta_{\theta}(p)$. Joint multifractal moments have been studied in more details in Ref. [25,26]. If $\Delta U_{\ell}$ and $\Delta \theta_{\ell}$ are independent random variables, one has $z(p, q)=\zeta_{\theta}(p)+\zeta_{u}(q)$; in the general case this is not verified due to couplings between the two fields. In the $(p, q)$ plane, there is one line, of equation $p=2 q$, which is particular, since it depends only on the passive scalar variance flux $\chi_{\ell}$ :

$$
\left\langle\left(\Delta \theta_{\ell}^{2} \Delta U_{\ell}\right)^{q / 3}\right\rangle \sim \ell^{\zeta_{m}(q)}
$$

where we denote $\zeta_{m}(q)=z(2 q / 3, q / 3)$. The dimensional relation $[16,18]$

$$
\chi_{\ell} \sim \frac{\Delta \theta_{\ell}^{2} \Delta U_{\ell}}{\ell}
$$

leads to:

$$
K_{\chi}(q)=q-\zeta_{m}(3 q)
$$

where $K_{\chi}(q)$ is the scaling exponent of the passive scalar flux (see $\left.[16,18]\right)$ :

$$
\left\langle\left(\chi_{\ell}\right)^{q}\right\rangle \sim \ell^{-K_{\chi}(q)}
$$

$K_{\chi}$ is a second characteristic function; it is nonlinear and concave, and the conservation of the flux leads to

$$
K_{\chi}(1)=0
$$

This relation for the flux corresponds also to Yaglom's 1949 exact relation 
involving passive scalar dissipation [27]:

$$
\left\langle\Delta \theta_{\ell}^{2} \Delta U_{\ell}\right\rangle=-\frac{4}{3} \chi \ell
$$

Equations (11) or (12) indicate that $\zeta_{m}(3)=1$ so that the mixed structure functions scaling exponent $\zeta_{m}(q)$ has the same "fixed point" as the velocity exponent $\zeta_{u}(q)$. These two curves are also identical for non-intermittent KOC turbulence. This explains why $\zeta_{u}(q)$ and $\zeta_{m}(q)$ have sometimes been compared. The first comparison (to our knowledge) was done by Schmitt et al. [18] for high Reynolds number atmospheric turbulence: the two curves were almost superposed until moment of order 5 . For larger moments, $\zeta_{m}(q)$ was slightly smaller than $\zeta_{u}(q)$. The same result was obtained using DNS data by Boratav and Pelz $\left(\operatorname{Pr}=1\right.$ and $\left.R_{\lambda}=141\right)$ for moments of order 2, 4 and 6 [19] and by Watanabe and Gotoh $\left(\operatorname{Pr}=1\right.$ and $\left.R_{\lambda}=427\right)$ [23]; in the latter case, mixed structure functions exponents have been estimated for integer moments and shown to be clearly different (and smaller) from velocity exponents only for moments larger than 4 . Using experimental data of the turbulent flow between coaxial rotating disks ( $\mathrm{Pr}=.7$ and $\left.R_{\lambda}=367\right)$, Pinton et al. [28] obtained identical scaling exponents (within experimental errors) for moments from 1 to 5 . Experimental data of the flow downstream of a cylinder analyzed by Lévêque et al [29] $\left(\operatorname{Pr}=.7\right.$ and $\left.R_{\lambda}=300\right)$ showed close values for moments less than 5 ; for larger moments, $\zeta_{m}(q)$ was slightly smaller than $\zeta_{u}(q)$. Mixed structure functions are also considered in $[30,20,31]$ for some orders of moments. Since in the following the connection between Eulerian and Lagrangian scaling exponents involves $\zeta_{m}(q)$, we give in Table 2 the values reported in the above mentioned papers, which are also represented in Fig. 2.

We note from Fig. 2 and Table 2 that the values obtained in [18] are close to most other published values up to moments of order 6 . Therefore in the last section, we take these values as representative of experimental estimates of $\zeta_{m}(q)$ until moments of order 6 .

The different studies mentioned here correspond to different Reynolds numbers, and include atmospheric observations, laboratory experiments for various types of flows (jets, rotating flows, homogeneous turbulence), and DNS data. Despite this variety of conditions, the scaling exponents are very stable and show, up to moments of order 5 , a quite low variability between different numerical estimates.

\subsection{Lagrangian case}

For the Lagrangian case, the situation is simpler, since Inoue's law (Eq.(4)) can be generalized to take into account intermittency using only one flux 
(written $\varphi_{\tau}$ in a Lagrangian framework), giving $\Delta \Theta_{\tau} \sim \varphi_{\tau}^{1 / 2} \tau^{1 / 2}$, and not a nonlinear product between two fluxes, as was the case for Eulerian passive scalar turbulence. Equation (4) has been generalized by Novikov, writing [32]:

$$
\left\langle\Delta \Theta_{\tau}^{q}\right\rangle \sim \tau^{\xi_{\theta}(q)}
$$

As for the Eulerian case, for a constant dissipation one obtains the "mean field" expression, neglecting intermittency: $\xi_{\theta}(q)=q / 2$. In this framework, the third order moment for the mixed Eulerian scaling exponent is analogous to the second order moment for the Lagrangian passive scalar: in case of intermittency $\xi_{\theta}(q)$ is nonlinear and concave, and the non-intermittent value is valid only for $q=2: \xi_{\theta}(2)=1$, indicating also that there is no intermittency correction for the power spectrum exponent.

The Eulerian exponents $\zeta_{\theta}(q)$ have been estimated experimentally for many years; to our knowledge, Lagrangian passive scalar exponents have been estimated only for marine turbulence with a relatively low number of points $[33,34]$ so that the resulting curve is close to $q / 2$. We provide in the following theoretical hypotheses leading to direct or parametric links between Lagrangian scaling exponents and Eulerian passive scalar and mixed velocitypassive scalar scaling exponents. The rest of the paper is devoted to this issue. The next section recalls and derives theoretical relations between these functions, corresponding to different simple or more realistic hypotheses linking space and time scales and Lagrangian and Eulerian statistics.

\section{Theoretical relations between Eulerian and Lagrangian scaling exponents}

\subsection{Different hypothesis to relate Eulerian and Lagrangian statistics and time and space}

As we have done elsewhere for the velocity [35], we introduce here two different hypotheses to relate Eulerian and Lagrangian statistics and obtain some relations for Eulerian and Lagrangian scaling exponents.

First, Tennekes and Lumley [6] have used ergodicity arguments for homogeneous and isotropic turbulence in a box, and obtained that the moments of an observable estimated in an Eulerian or Lagrangian frame are identical. This was developed by Borgas [36] for the moments of the dissipation field $\epsilon$. Recently, this was extended to codimensions (i.e. singularity spectra) of Eulerian and Lagrangian velocity fluctuations [37-39].

In a phenomenological way, it can also be done for fluxes, and using $\varphi_{\tau}$ intro- 
duced above, we obtain the hypothesis of equality for Eulerian and Lagrangian moments:

$$
\left\langle\chi_{\ell}^{q}\right\rangle=\left\langle\varphi_{\tau}^{q}\right\rangle \quad \text { Hyp.A }
$$

This can be used to relate Eulerian and Lagrangian scaling exponents, if a dimensional relation between space and time is available.

In the same spirit, some other studies of Lagrangian intermittent velocity [4042] have introduced another argument: they consider the velocity advecting Lagrangian trajectories as a superposition of different velocity contributions coming from different eddies having different characteristic times. After a time $\tau$ the fastest eddies, of scale smaller than $\ell$, are decorrelated so that at leading order, they assume that $\Delta V_{\tau} \sim \Delta U_{\ell}$. The same approach for the passive scalar associated to these turbulent eddies leads to a second hypothesis to relate Eulerian and Lagrangian statistics:

$$
\Delta \Theta_{\tau} \sim \Delta \theta_{\ell} \quad \text { Hyp.B }
$$

A priori this hypothesis and the previous one (Eq. (14)) are different and may lead to different scaling laws; this will be considered below.

This is not enough to relate Eulerian and Lagrangian scaling exponents; a relation linking space and time is also necessary. There are two possibilities. First, noting $U=\ell / \tau$, Kolmogorov's relation gives, neglecting the intermittency of the dissipation field:

$$
\ell^{2} \sim \tau^{3} \quad \text { Hyp. } \alpha
$$

This is also Richardson's dispersion law [43] for non-intermittent turbulence. This is equivalent to assume that the dissipation, which is proportional to $\ell^{2} / \tau^{3}$, has a constant value.

On the other hand, taking into account intermittency for the Eulerian velocity, one can write $\Delta U_{\ell} \sim \ell^{h_{u}}$, where $h_{u}$ is the singularity of the velocity fluctuations [44]. Since we have also $\Delta U_{\ell} \sim \ell / \tau$, this gives [40-42]:

$$
\tau \sim \ell^{1-h_{u}} \quad \text { Hyp. } \beta
$$

This is a local random space-time relation and corresponds to take into account the intermittency of the dissipation.

To relate Eulerian and Lagrangian scaling exponents, one must choose one of the statistical relations (Hypothesis A or B) and one of the time-scale relations (Hypothesis $\alpha$ or $\beta$ ). We precise below the predictions corresponding to all these four possibilities. 


\subsection{Two simple relations to relate $\xi_{\Theta}(q)$ to $\zeta_{\theta}(q)$ or $\zeta_{m}(q)$}

The first and simplest choice we consider is to assume the "characteristic time" relation and the non-intermittent space-time relation (Hypotheses $B-\alpha$ ), providing, using Eqs.(5) and (13):

$$
\xi_{\Theta}(q)=\frac{3}{2} \zeta_{\theta}(q) \quad \text { CaseI }
$$

With this approach the normalization condition $\xi_{\Theta}(2)=1$ is not met unless $\zeta_{\theta}(2)$ is exactly $2 / 3$. In fact, considering the usual values of $\zeta_{\theta}(2)$ close to $2 / 3$, the value of $\xi_{\Theta}(2)$ will be close to 1 .

The second simplest choice is to take the "ergodic" relation and the nonintermittent space-time relation (Hypotheses $A-\alpha$ ). Let us first introduce $K_{\varphi}(q)$, the Lagrangian scaling exponent of the passive scalar dissipation flux, defined as:

$$
\left\langle\varphi_{\tau}^{q}\right\rangle \sim \tau^{-K_{\varphi}(q)}
$$

Then Hypotheses A- $\alpha$, together with Eqs. (10) and (19) give:

$$
K_{\varphi}(q)=\frac{3}{2} K_{\chi}(q)
$$

Using Eq.(9) and since $K_{\varphi}(q)=q-\xi_{\Theta}(2 q)$ (coming from the fact that the flux may be written $\left.\varphi_{\tau} \sim \Delta \Theta_{\tau}^{2} / \tau\right)$, this leads to:

$$
\xi_{\Theta}(q)=\frac{3}{2} \zeta_{m}\left(\frac{3 q}{2}\right)-\frac{q}{4} \quad \text { CaseII }
$$

We can check that for $q=2$, we obtain as expected $\xi_{\Theta}(2)=1$ since $\zeta_{m}(3)=1$. This is a simple relation but, a priori, case II leads to a different prediction than case I. Indeed the latter involves Eulerian mixed velocity-temperature scaling exponent $\zeta_{m}(q)$, whereas the former involves directly the Eulerian temperature scaling exponent $\zeta_{\theta}(q)$ itself.

\subsection{Intermittency in the space-time relation and a third proposal to relate $\zeta_{m}(q)$ and $\xi_{\Theta}(q)$}

We consider now another case, which is also more complete, since it involves intermittency in the relation between space and time: Hypotheses $A-\beta$. This involves a Legendre transform which is classically used in the multifractal framework and is recalled for convenience in Appendix 1.

Using Hypothesis A, we write the moments in two different manners. On the one hand, we have Eq.(19). On the other hand, we use Eq.(8) and write, 
(denoting $h_{\theta}$ and $h_{u}$ the singularities of the Eulerian temperature and velocity increments):

$$
\begin{aligned}
\left\langle\chi_{\ell}^{q}\right\rangle & \sim \frac{\left\langle\Delta \theta_{\ell}^{2 q} \Delta U_{\ell}^{q}\right\rangle}{\ell^{q}} \\
& \sim \int_{\left(h_{\theta}, h_{u}\right)} \ell^{2 q h_{\theta}+q h_{u}+c\left(h_{\theta}, h_{u}\right)-q} d\left(h_{\theta}, h_{u}\right) \\
& \sim \int_{\left(h_{\theta}, h_{u}\right)} \tau^{\frac{2 q h_{\theta}+q h_{u}+c\left(h_{\theta}, h_{u}\right)-q}{1-h_{u}}} d\left(h_{\theta}, h_{u}\right)
\end{aligned}
$$

where in the last line we have used Hypothesis $\beta$ which relates locally time and space, through the introduction of the velocity singularity $h_{u}$. Using Eqs.(14) and (19), we thus have:

$$
-K_{\varphi}(q)=\min _{\left(h_{\theta}, h_{u}\right)}\left(\frac{2 q h_{\theta}+q h_{u}+c\left(h_{\theta}, h_{u}\right)-q}{1-h_{u}}\right)
$$

Here the minimum value is not searched for across the full plane $\left(h_{\theta}, h_{u}\right)$; since the moments are proportional, the singularities are also proportional (see Appendix 2) and we have $h_{\theta}=h_{u} / 2$. The minimum value is thus rather obtained along a cut through a line of equation $h_{\theta}=h_{u} / 2$. Let us denote $c\left(h_{\theta}, h_{u}\right)$ the codimension of the mixed temperature-velocity moments and $c_{1}\left(h_{u}\right)=$ $c\left(h_{u} / 2, h_{u}\right)$. Then, using the result presented in Appendix 2 in Eq.(38), we have the following relation involving the mixed moments $\zeta_{m}(3 q)=z(2 q, q)$ :

$$
\left\{\begin{array}{l}
\zeta_{m}(3 q)=2 q h_{u}+c_{1}\left(h_{u}\right) \\
q=-\frac{1}{2} c_{1}^{\prime}\left(h_{u}\right)
\end{array}\right.
$$

This relation provides a one-to-one relation between singularities $h_{u}$ and order of moments $q$. This can be used to evaluate Eq.(22). We write $-K_{\varphi}(q)=$ $\min _{h_{u}}\left\{H_{q}\left(h_{u}\right)\right\}$, with $H_{q}$ given by:

$$
H_{q}\left(h_{u}\right)=\frac{2 q h_{u}+c_{1}\left(h_{u}\right)-q}{1-h_{u}}
$$

As we have done elsewhere for similar expressions [35,45], this is solved by equating $H_{q}^{\prime}\left(h_{0}\right)=0$ for a particular singularity value denoted $h_{0}$, which is associated to an order of moments $q_{0}$. The condition $H_{q}^{\prime}\left(h_{0}\right)=0$ gives:

$$
q+c_{1}^{\prime}\left(h_{0}\right)\left(1-h_{0}\right)+c_{1}\left(h_{0}\right)=0
$$

and with the introduction through Eq.(23) of the moment of order $q_{0}$ associated to $h_{0}$, this simplifies into:

$$
\zeta_{m}\left(3 q_{0}\right)=2 q_{0}-q
$$


Equation (26), together with another use of the Legendre transform (23), gives the Lagrangian exponent:

$$
\begin{aligned}
-K_{\varphi}(q)=H_{q}\left(h_{0}\right) & =\frac{2 q h_{0}+c_{1}\left(h_{0}\right)-q}{1-h_{0}} \\
& =\frac{2 q h_{0}+2 q_{0}-2 q-2 q_{0} h_{0}}{1-h_{0}} \\
& =2 q_{0}-2 q
\end{aligned}
$$

Using again $K_{\varphi}(q)=q-\xi_{\Theta}(2 q)$ this gives finally the following parametric relation between Lagrangian passive scalar scaling exponents and the Eulerian mixed moments:

$$
\left\{\begin{array}{l}
\xi_{\Theta}(q)=\zeta_{m}\left(q_{0}\right) \quad \text { Case III } \\
\frac{q}{2}=\frac{2 q_{0}}{3}-\zeta_{m}\left(q_{0}\right)
\end{array}\right.
$$

This relation provides $\xi_{\Theta}(q)$ when the fonction $\zeta_{m}(q)$ is known, the second line giving the link between $q$ and $q_{0}$. We can check that for $q_{0}=3$, the second line gives $q=2$ and the first one, $\xi_{\Theta}(2)=\zeta_{m}(3)=1$ as expected.

\subsection{A fourth relation}

We now consider the fourth case, corresponding to Hypotheses $B-\beta$. This gives directly, following a path analogous to case III:

$$
\begin{aligned}
\tau^{\xi_{\Theta}(q)} \sim\left\langle\Delta \Theta_{\tau}^{q}\right\rangle & \sim\left\langle\Delta \theta_{\ell}^{q}\right\rangle \\
& \sim \int \ell^{q h_{\theta}+c\left(h_{\theta}\right)} d p\left(h_{\theta}\right) \\
& \sim \int \tau^{\frac{q h_{\theta}+c\left(h_{\theta}\right)}{1-h_{u}}} d p\left(h_{\theta}\right)
\end{aligned}
$$

This then gives the following Lagrangian scaling exponent:

$$
\xi_{\Theta}(q)=\min _{\left(h_{\theta}, h_{u}\right)}\left(\frac{q h_{\theta}+c\left(h_{\theta}\right)}{1-h_{u}}\right)
$$

In this expression, the min is taken not on the whole $\left(h_{\theta}, h_{u}\right)$ plane, but on a set corresponding to joint $\left(h_{\theta}, h_{u}\right)$ singularities associated to an actual physical situation. Without further hypotheses on the statistical links between $h_{\theta}$ and $h_{u}$, there is no way to go further. For non-intermittent Kolmogorov turbulence, $h_{u}=1 / 3$ is not random and we recover directly case I: $\xi_{\Theta}(q)=\frac{3}{2} \zeta_{\theta}(q)$. If $h_{\theta}$ 
and $h_{u}$ are independent, we may also simplify Eq.(28):

$$
\begin{aligned}
\xi_{\Theta}(q) & =\frac{\min _{h_{\theta}}\left\{q h_{\theta}+c\left(h_{\theta}\right\}\right.}{\max _{h_{u}}\left\{1-h_{u}\right\}} \\
& =\frac{\zeta_{\theta}(q)}{1-h_{\min }}
\end{aligned}
$$

where $h_{\text {min }}$ denotes the smallest among all the $h_{u}$ singularities. Independence between $h_{\theta}$ and $h_{u}$ is of course very unlikely to be met in real situations.

Another possibility is to consider a situation of conditional expectation between velocity and temperature singularities as follows:

$$
\left\langle h_{u} \mid h_{\theta}\right\rangle=2 h_{\theta}
$$

This is in agreement with the relation obtained in another context for the conditional expectation of velocity increment versus temperature increment $[46,47]$. Within this framework, we assume here that $h_{u}$ can be replaced by $2 h_{\theta}$ in Eq.(28), leading to

$$
\xi_{\Theta}(q)=\min _{h_{\theta}}\left(\frac{q h_{\theta}+c\left(h_{\theta}\right)}{1-2 h_{\theta}}\right)
$$

This can be solved as done above, to provide an explicit relation giving $\xi_{\Theta}(q)$ : we introduce a moment of order $q_{0}$ associated to the singularity $h_{0}$ minimizing the expression given in Eq.(30). After using twice the Legendre transform Eq.(34) this gives:

$$
\left\{\begin{array}{l}
\xi_{\Theta}(q)=\zeta_{\theta}\left(q_{0}\right) \\
q=q_{0}-2 \zeta_{\theta}\left(q_{0}\right)
\end{array} \quad\right. \text { Case IV }
$$

This provides a direct parametric relation between Eulerian and Lagrangian passive scalar scaling exponents. We may note that for $q=2$ we know that $\xi_{\Theta}(2)=1$ since there is no intermittency correction for the second moment. The associated moment is $q_{0}=4$ and this leads to $\zeta_{\theta}(4)=1$. There is up to now no theory predicting such exact relation for the fourth Eulerian moment; however, this value is compatible with available estimates. Furthermore, the average result provided in Table 1 indicates $\zeta_{\theta}(4)=.99 \pm .05$, which is very close to this exact value. This may be a point in favor of this fourth relation.

\section{Comparison of the different predictions}

We compare here the predictions provided by case I to IV. For case I and case IV, we take here for Eulerian scaling exponents the average values estimated above (Table 1), which are a rather good compromise between many 
published values (see Fig. 1). For case II and case III, providing a prediction for Lagrangian passive scalars as a relation to the mixed Eulerian exponents, we take for $\zeta_{m}(q)$ the values estimated in [18], which are close to other values reported in the literature (see Table 2 and Fig. 2) for moments up to about 6 . These different predictions are displayed in Fig. 3. It is interesting to note that case I to III are very close for low orders moments, which can be understood by the fact that intermittency effects are expected to become important mainly for high order moments. This does not mean that these cases are almost identical: the underlying hypotheses are clearly different, and questions linked to higher moments (high order correlations, return times, large fluctuations) have quite different output since scaling exponents are different. Furthermore, the deviation from linearity is stronger for case I and case II, which may indicate that to take into account intermittency in the space-time relation reduces in fact the apparent intermittency of the Lagrangian estimates. The fourth prediction is quite far from the others, except the common point $\zeta_{\theta}(2)=1$. This may be the consequence of the additional hypothesis which was needed to obtain case IV prediction.

Let us discuss the different predictions which are provided here. A priori the local space-time relation influenced by intermittency in velocity fluctuations is the more realistic (Hypothesis $\beta$ ). The predicitons corresponding to case I and case II are thus mentioned mainly for illustration purposes. The predictions associated to case III and case IV are the more realistic, since they use an intermittent space-time relation. A validation using experimental or numerical data will be needed to choose between these cases. In the near future, when experimental or numerical Lagrangian passive scalar data will become available, the first validation will involve testing case IV versus case III, since the scaling exponents associated to each case are very different. If case III is the closer to data, then much more data points will be needed to clearly separate the predictions associated to case I, II or III. This may be provided by DNS studies.

\section{Conclusion}

In another paper [35] devoted to a comparison of different relations between Eulerian and Lagrangian scaling exponents for velocity turbulence, we have been able to compare predictions to experimental and DNS results. In the present paper dealing with the same issue concerning passive scalars, comparisons with experimental or numerical data is not yet possible. We have proposed here four new predictions for $\xi_{\theta}(q)$, the passive scalar Lagrangian scaling exponent, expressed as function of $\zeta_{\theta}(q)$ and $\zeta_{m}(q)$, the Eulerian passive scalar exponent and mixed velocity-temperature scaling exponents respectively. Among these four explicit relations (case I to IV) only case III and IV 
seem realistic, since they take into account the local variability of space and time relation for intermittent velocity. The three first outputs are quite close to each other for low orders of moments $(q \leq 4)$, whereas they become distinguishable only for larger moments. The last prediction (case IV) appears to provide a very different $\xi_{\Theta}(q)$ function. The fact that scaling exponents are close does not mean that these cases are almost identical: the underlying hypotheses are different, and questions linked to higher moments, such as higher correlations, have quite different output since larger scaling exponents are different.

It will be interesting to be able to check these results with experiments. Let us note that, while DNS lagrangian results seem accessible, experimental estimates are not easy to realize at small scales. Since the scaling exponents associated to case III and case IV are very different, we can expect that DNS studies in the near future may be able to confirm one of these relations. On the experimental side, we plan in future studies to associate small-scale high frequency temperature probes with a small floating device on the surface of water (buoy) to study passive scalar Lagrangian marine turbulence and estimate its scaling exponents.

\section{Appendix 1}

We recall here the main properties of the multifractal formalism, including the codimension function and the Legendre transform between the moment function $\zeta(q)$ and the codimension function $c(h)$.

In this framework one may characterize the velocicty fluctuations $\Delta U_{\ell}$ at scale $\ell$ through the singularities $h$ and their codimension $c(h)[44,48,9,12]$ :

$$
\Delta U_{\ell} \sim \ell^{h} ; \quad p\left(\Delta U_{\ell}\right) \sim \ell^{c(h)}
$$

Here the codimension is used instead of the more frequent dimension $f(h)=$ $d-c(h)$, where $d$ is the dimension of the space $(d=1$ or $d=3$ in most studies), and $p\left(\Delta U_{\ell}\right)$ is the probability density of velocity fluctuations. The moments write:

$$
\begin{aligned}
\left\langle\Delta U_{\ell}^{q}\right\rangle & =\int \Delta U_{\ell}^{q} p\left(\Delta U_{\ell}\right) \\
& \sim \int \ell^{q h+c(h)} d p(h) \sim \ell^{\zeta(q)}
\end{aligned}
$$

where $\zeta(q)$ is the scale invariant moment function characterizing the fluctuations. Using a saddle point argument [44], this gives the classical Legendre 
transform between $\zeta(q)$ and $c(h)$ :

$$
\zeta(q)=\min _{h}\{q h+c(h)\}
$$

This can also be written in the following way, emphasizing the one-to-one relation between orders of moment $q$ and singularities $h$ :

$$
\left\{\begin{array}{l}
q h+c(h)=\zeta(q) \\
q=-c^{\prime}(h)
\end{array}\right.
$$

In this way each singularity is associated to a unique order of moments.

\section{Appendix 2}

We provide here the basic results obtained for joint moments of a multifractal field. We recall the expression of the Legendre transform and discuss a particular case.

Let us consider joint moments of two multifractal field of the form $\left\langle\Delta X_{\ell}^{p} \Delta Y_{\ell}^{q}\right\rangle$. These fields are characterized by singularities $h_{x}$ and $h_{y}$ :

$$
\begin{aligned}
\Delta X_{\ell} & \sim \ell^{h_{x}} \\
\Delta Y_{\ell} & \sim \ell^{h_{y}}
\end{aligned}
$$

We also introduce the joint moment scaling exponent $z(p, q)$ and the joint codimension $c\left(h_{x}, h_{y}\right)$ :

$$
\begin{aligned}
\left\langle\Delta X_{\ell}^{p} \Delta Y_{\ell}^{q}\right\rangle & \sim \ell^{z(p, q)} \\
p\left(\Delta X_{\ell}, \Delta Y_{\ell}\right) & \sim \ell^{c\left(h_{x}, h_{y}\right)}
\end{aligned}
$$

Using the usual saddle-point approximation, these functions are related through a double-integral:

$$
z(p, q)=\min _{\left(h_{x}, h_{y}\right)}\left\{p h_{x}+q h_{y}+c\left(h_{x}, h_{y}\right)\right\}
$$

This can also be written as [25]:

$$
\left\{\begin{array}{l}
z(p, q)=p h_{x}+q h_{y}+c\left(h_{x}, h_{y}\right) \\
p=-\frac{\partial c}{\partial h_{x}}\left(h_{x}, h_{y}\right) \\
q=-\frac{\partial c}{\partial h_{y}}\left(h_{x}, h_{y}\right)
\end{array}\right.
$$


This relation can also be inverted to provide:

$$
\left\{\begin{array}{l}
z(p, q)=p h_{x}+q h_{y}+c\left(h_{x}, h_{y}\right) \\
h_{x}=\frac{\partial z}{\partial p}(p, q) \\
h_{y}=\frac{\partial z}{\partial q}(p, q)
\end{array}\right.
$$

Let us now consider a "linear cut" in the $(p, q)$ plane, and choose $p=a q$ with $a>0$. There is only one degree of freedom in the moments, and hence one degree of freedom for singularities which are thus also proportional: Eq.(37) gives $h_{x}=\frac{1}{a} h_{y}$. Let us note $z_{a}(q)=z(a q, q)$. Relation (37) then becomes

$$
\left\{\begin{array}{l}
z_{a}(q)=2 q h_{y}+c\left(\frac{1}{a} h_{y}, h_{y}\right) \\
h_{y}=\frac{\partial z}{\partial q}(a q, q)=\frac{1}{2} z_{a}^{\prime}(q)
\end{array}\right.
$$

coming from the fact that $z_{a}^{\prime}(q)=a \frac{\partial z}{\partial p}(a q, q)+\frac{\partial z}{\partial q}(a q, q)=2 \frac{\partial z}{\partial q}(a q, q)$. This is thus analogous to a 1-D multifractal with $h=2 h_{y}$ and $c(h)=c\left(\frac{1}{a} h_{y}, h_{y}\right)$.

\section{References}

[1] Z. Warhaft, Annual Rev. Fluid Mech. 32, 203 (2000).

[2] S. Pope, Turbulent flows (Cambridge University Press, 2000).

[3] A. N. Kolmogorov, C. R. Acad. Sci. URSS 30, 301 (1941).

[4] A. M. Obukhov, Izv. Akad. Nauk. SSSR Geogr. Geofiz. 13, 58 (1949).

[5] S. Corrsin, J. Appl. Phys. 22, 469 (1951).

[6] H. Tennekes and J. L. Lumley, A First Course in Turbulence (MIT Press, 1972).

[7] L. D. Landau and E. M. Lifshitz, Fluid Mechanics (MIR, 1944), first russian edition ed.

[8] E. Inoue, J. Meteorol. Soc. Japan 29, 246 (1952).

[9] U. Frisch, Turbulence; the legacy of A. N. Kolmogorov (Cambridge University Press, 1995).

[10] R. Livi and A. Vulpiani, eds., L’héritage de Kolmogorov en physique (Belin, Paris, 2003).

[11] T. Bohr, M. H. Jensen, G. Paladin, and A. Vulpiani, Dynamical systems approach to turbulence (Cambridge University Press, 1998).

[12] D. Schertzer, S. Lovejoy, F. Schmitt, Y. Chigirinskaya, and D. Marsan, Fractals 5, 427 (1997). 
[13] A. Arnéodo, C. Baudet, F. Belin, R. Benzi, B. Castaing, B. Chabaud, R. Chavarria, S. Ciliberto, R. Camussi, F. Chilla, et al., Europhys. Lett. 34, 411 (1996).

[14] R. H. Kraichnan, Phys. Rev. Lett. 72, 1016 (1994).

[15] G. Falkovich, K. Gawedzki, and M. Vergassola, Rev. Mod. Phys. 73, 913 (2001).

[16] R. A. Antonia, E. J. Hopfinger, Y. Gagne, and F. Anselmet, Phys. Rev. A 30, 2704 (1984).

[17] G. Ruiz-Chavarria, C. Baudet, and S. Ciliberto, Physica D 99, 369 (1996).

[18] F. G. Schmitt, D. Schertzer, S. Lovejoy, and Y. Brunet, Europhys. Lett. 34, 195 (1996).

[19] O. N. Boratav and R. B. Pelz, Phys. Fluids 10, 2122 (1998).

[20] G. Xu, R. A. Antonia, and S. Rajagopalan, Europhys. Lett. 49, 452 (2000).

[21] F. Moisy, H. Willaime, J. S. Andersen, and P. Tabeling, Phys. Rev. Lett. 86, 4827 (2001).

[22] A. Gylfason and Z. Warhaft, Phys. Fluids 16, 4012 (2004).

[23] T. Watanabe and T. Gotoh, New J. Phys. 6, 40 (2004).

[24] R. A. Antonia and C. W. V. Atta, J. Fluid Mech. 67, 273 (1975).

[25] C. Meneveau, K. R. Sreenivasan, P. Kailasnath, and M. S. Fan, Phys. Rev. A 41, 894 (1990).

[26] L. Seuront and F. G. Schmitt, Deep Sea Res. II 52, 1308 (2005).

[27] A. M. Yaglom, Dokl. Akad. Nauk. SSSR 69, 743 (1949).

[28] J.-F. Pinton, F. Plaza, L. Danaila, P. L. gal, and F. Anselmet, Physica D 122, 187 (1998).

[29] E. Leveque, G. Ruiz-Chavarria, C. Baudet, and S. Ciliberto, Phys. Fluids 11, 1869 (1999).

[30] R. Rao, Phys. Rev. E 59, 1727 (1999).

[31] L. Mydlarski, J. Fluid Mech. 475, 173 (2003).

[32] E. A. Novikov, Phys. Fluids A 1, 326 (1989).

[33] L. Seuront, F. Schmitt, D. Schertzer, Y. Lagadeuc, and S. Lovejoy, Nonlinear Proc. Geophys. 3, 236 (1996).

[34] L. Seuront and F. G. Schmitt, Geophys. Res. Lett. 31, L03306 (2004).

[35] F. G. Schmitt, Physica A (submitted).

[36] M. S. Borgas, Phil. Trans. R. Soc. Lond. A 342, 379 (1993). 
[37] N. Mordant, O. Michel, P. Metz, and J.-F. Pinton, Phys. Rev. Lett. 87, 21 (2001).

[38] L. Chevillard, S. G. Roux, E. Léêque, N. Mordant, J.-F. Pinton, and A. Arnéodo, Phys. Rev. Lett. 91, 214502 (2003).

[39] L. Chevillard, Description multifractale unifiée du phénomène d'intermittence en turbulence Eulérienne et Lagrangienne ( $\mathrm{PhD}$ Thesis, University of Bordeaux I available online at http://tel.ccsd.cnrs.fr, 2004).

[40] G. Boffetta, F. D. Lillo, and S. Musacchio, Phys. Rev. E 66, 066307 (2002).

[41] L. Biferale, G. Boffetta, A. Celani, B. J. Devenish, A. Lanotte, and F. Toschi, Phys. Rev. Lett. 93, 064502 (2004).

[42] L. Biferale, G. Boffetta, A. Celani, A. Lanotte, and F. Toschi, Phys. Fluids 17, 021701 (2005).

[43] L. F. Richardson, Proc. Roy. Soc. London A 110, 709 (1926).

[44] G. Parisi and U. Frisch, in Turbulence and Predictability in Geophysical Fluid Dynamics and Climate Dynamics, edited by M. Ghil, R. Benzi, and G. Parisi (North Holland, Amsterdam, 1985), pp. 84-88.

[45] F. G. Schmitt, Phys. Lett. A 342, 448 (2005).

[46] Y. G. Sinai and V. Yakhot, Phys. Rev. Lett. 63, 1962 (1989).

[47] S. Vaienti, M. Ould-Rouis, F. Anselmet, and P. L. Gal, Physica D 73, 99 (1994).

[48] D. Schertzer and S. Lovejoy, J. Geophys. Res. 92, 9692 (1987). 
Table 1

Average values of $\zeta_{\theta}(q)$ with error bars, estimated from several published estimates [16-23].

\begin{tabular}{cc}
$\mathrm{q}$ & $\zeta_{\theta}(q)$ \\
\hline 1 & $.365 \pm .015$ \\
2 & $.65 \pm .03$ \\
3 & $.85 \pm .05$ \\
4 & $.99 \pm .05$ \\
5 & $1.10 \pm .05$ \\
6 & $1.20 \pm .08$ \\
7 & $1.30 \pm .1$ \\
8 & $1.40 \pm .12$
\end{tabular}

Table 2

Some recent experimental estimations for $\zeta_{m}(q)$. A: Large $R e$ : atmospheric data [18]; B: DNS data, $\operatorname{Pr}=1$ and $R_{\lambda}=141$ [19]; C: Experimental data, coaxial rotating disks, $\operatorname{Pr}=.7$ and $R_{\lambda}=367$ [28]; D: Experimental data, downstream of a cylinder, $\operatorname{Pr}=.7$ and $R_{\lambda}=300$ [29]; E: DNS data, $\operatorname{Pr}=1$ and $R_{\lambda}=427$ [23]; F: Experimental data, round jet, $\operatorname{Pr}=.7$ and $R_{\lambda}=550$ [20]; G: Experimental data, grid turbulence, $\operatorname{Pr}=.7$ and $R_{\lambda}=582$ [31].

\begin{tabular}{cccccccc}
$\mathrm{q}$ & $\mathrm{A}$ & $\mathrm{B}$ & $\mathrm{C}$ & $\mathrm{D}$ & $\mathrm{E}$ & $\mathrm{F}$ & $\mathrm{G}$ \\
\hline 0.5 & 0.21 & & 0.19 & & & & \\
1 & 0.39 & & 0.37 & 0.39 & 0.38 & & \\
1.5 & 0.56 & & 0.54 & & & & \\
2 & 0.72 & 0.72 & 0.70 & 0.73 & 0.70 & & \\
2.5 & 0.87 & & 0.85 & & & & \\
3 & 1 & 1 & 1 & 1 & 0.96 & 1.06 & 1 \\
3.5 & 1.12 & & 1.14 & & & & \\
4 & 1.24 & 1.25 & 1.27 & 1.23 & 1.19 & & \\
4.5 & 1.35 & & 1.39 & & & & \\
5 & 1.45 & & 1.50 & 1.43 & & & \\
6 & 1.65 & 1.83 & & 1.61 & 1.55 & 1.83 & 1.52 \\
7 & 1.83 & & & 1.80 & & & \\
8 & 2.00 & & & 1.95 & 1.86 & &
\end{tabular}




\section{$\zeta_{\theta}(q)$}

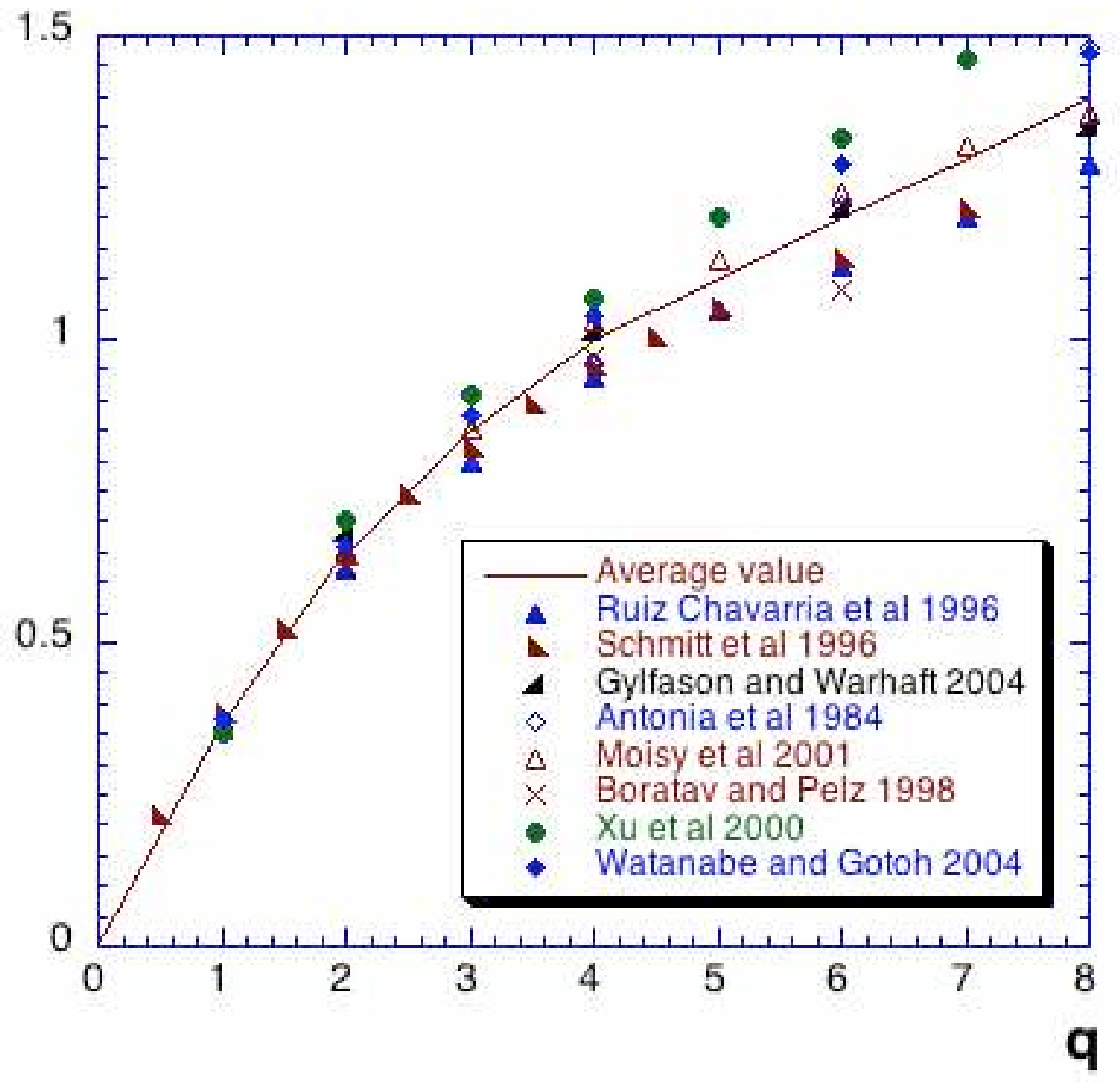

Fig. 1. The passive scalar Eulerian scaling exponent function $\zeta_{\theta}(q)$ estimated by various authors, and with an average fit (see Table 1). 


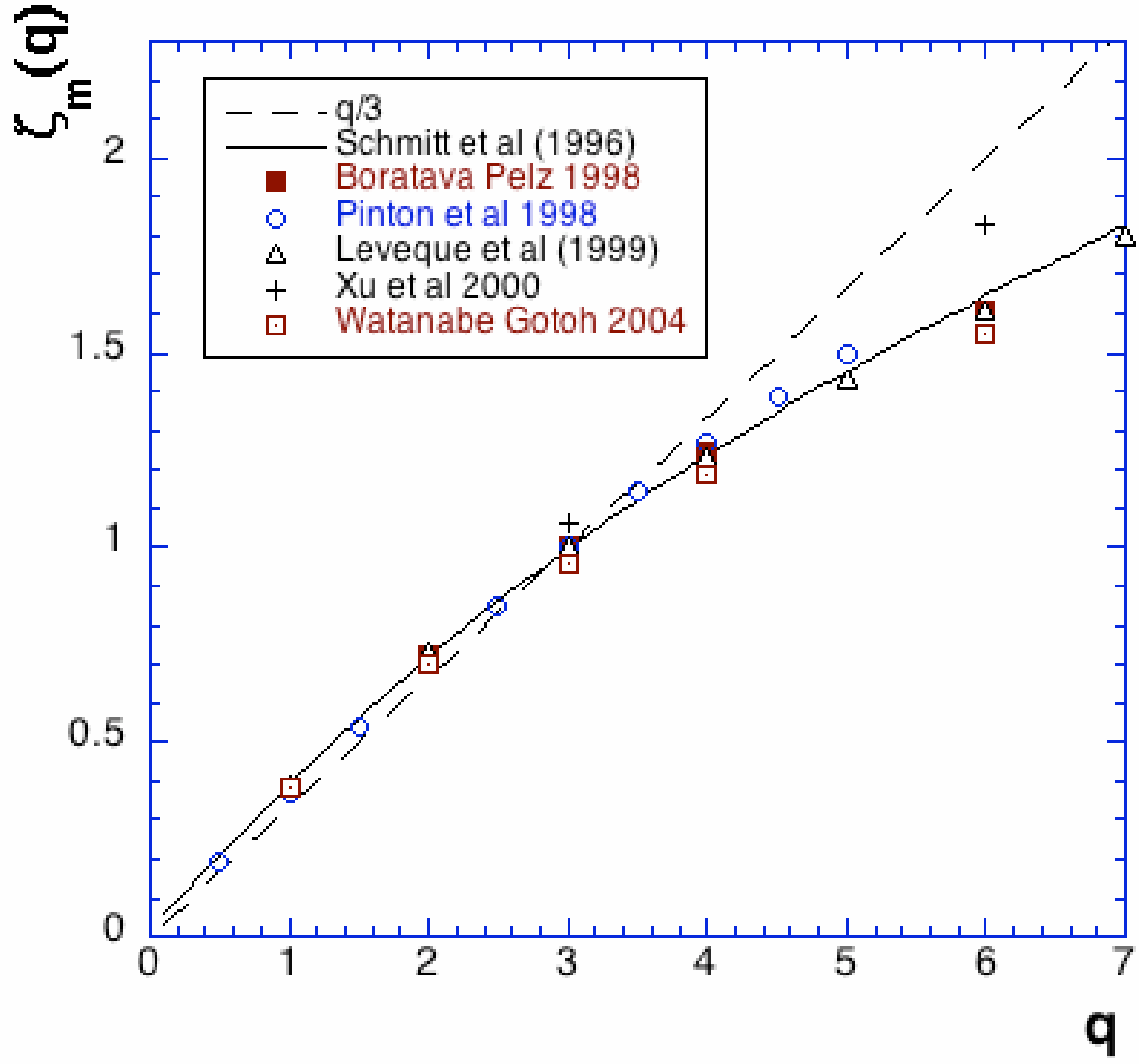

Fig. 2. The mixed velocity-temperature Eulerian scaling exponent function $\zeta_{m}(q)$ estimated by various authors. 


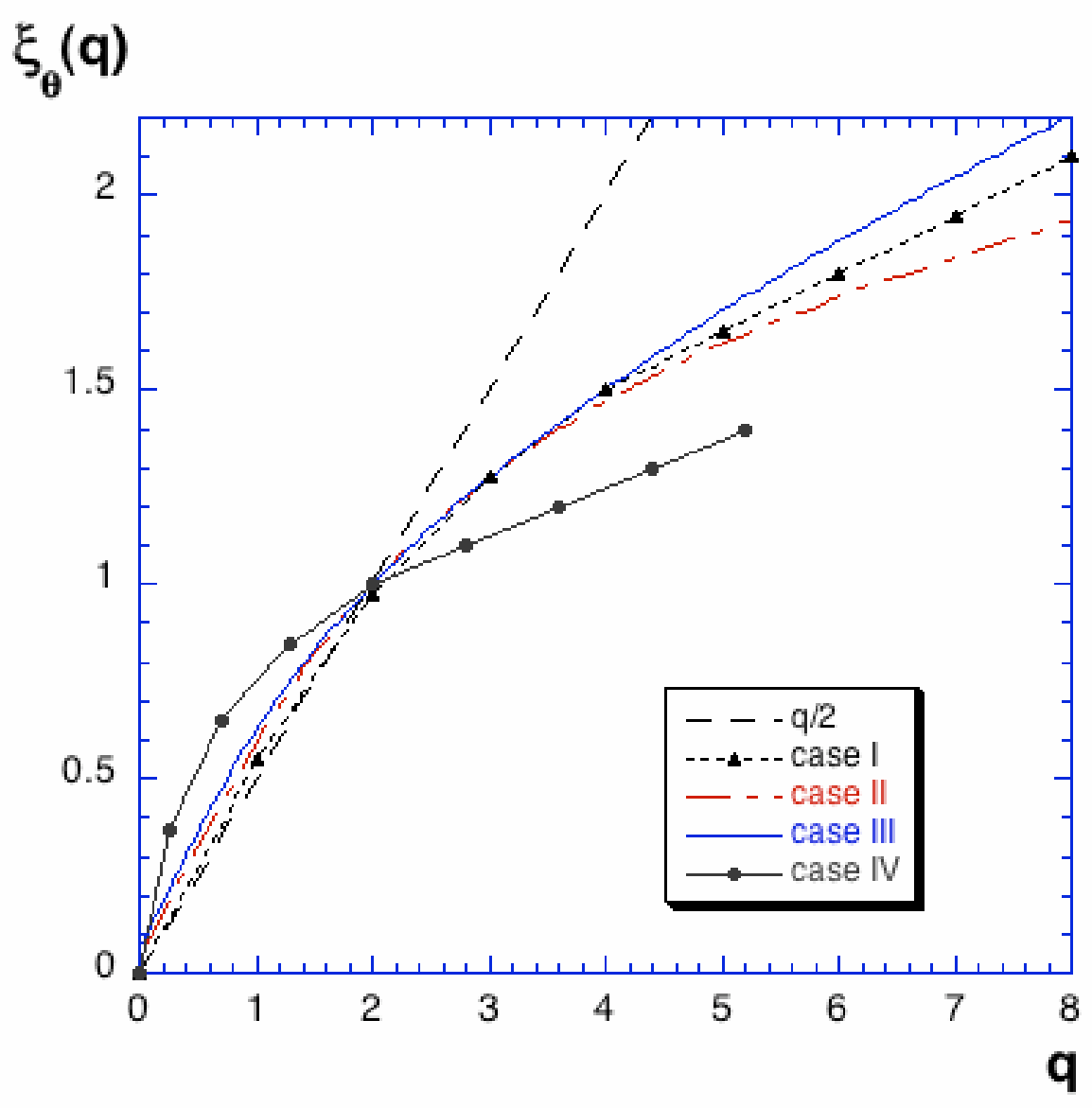

Fig. 3. Four different predictions for the Lagrangian passive scalar scaling exponent $\xi_{\Theta}(q)$. Case I and IV curves are obtained through experimental estimates of $\zeta_{\theta}(q)$, whereas case II and case III curves are obtained through experimental estimates of $\zeta_{m}(q)$. 